\title{
Thesis
}

\section{Enhancing the management of resource-aware business processes}

\author{
Cristina Cabanillas \\ Institute for Information Business, Vienna University of Economics and Business, Vienna, Austria \\ E-mail: cristina.cabanillas@wu.ac.at
}

\begin{abstract}
The resource perspective has received much less attention than other business process (BP) perspectives, e.g., control flow. This thesis focuses on human resource management in BPs, and addresses challenges related to resource specification and resource analysis. In particular, it introduces novel techniques for resource specification, which rely on a new resource selection language called RAL; and it provides automated support for a set of analysis operations at design time and at run time based on the formal semantics of RAL defined in description logics (DLs), by leveraging existing DL reasoners. All the contributions have been validated.

Keywords: Analysis operation, business process management, description logics, RAL, resource specification, resource analysis
\end{abstract}

\section{Motivation}

Providing support for Business Process Management (BPM) requires taking into account several perspectives throughout the entire BPM lifecycle. The BP resource perspective has received less attention than others such as control flow, despite resources are a key element in the correct execution of BP activities. This thesis [1] focuses on human resource ${ }^{1}$ management in BPs, specifically on resource specification, and on design-time (DT) and run-time (RT) resource analysis. Resource specification in BPs refers to the assignment of people to activities in which they can be involved, and resource analysis in BPs copes with reasoning on resource-aware BP models (i.e., BP models with information about resources) to infer information from them in order to understand how resources are involved in them.

The solutions for resource specification must be traceable to the concepts defined in the organisational model of the company; expressive, to define a wide variety of resource selection conditions; and flexible, to allow the user to decide how the resource specification is going to be bound to a resource-unaware BP model. A study of twenty-one approaches showed that,

\footnotetext{
${ }^{1}$ From now on, resource for the sake of brevity.
}

although most of them are traceable, more than $50 \%$ do not exceed a medium level of expressiveness, and evidence about binding flexibility was not found in any approach.

Regarding analysis, seven analysis operations related to the relation between people and activities (a.k.a. person-activity operations), and four analysis operations related to the permissions people have to access the information handled in BPs (a.k.a. persondata operations), were identified. A study of seven proposals considering resource analysis concluded that only one person-activity operation is supported in all cases, and only one approach offers support at DT (i.e., considering only static information modelled in the BP diagram) and at RT (i.e., considering also dynamic execution information). Another person-activity operation has often been addressed at DT. Support for the rest of operations is missing.

Therefore, the goal of this work was to develop traceable, expressive and flexible techniques to assign resources to BPs, and to develop mechanisms for the automation of the eleven analysis operations at DT and at RT.

\section{Contributions}

The approach developed to address the previous goals is depicted in Fig. 1 and summarised below. 


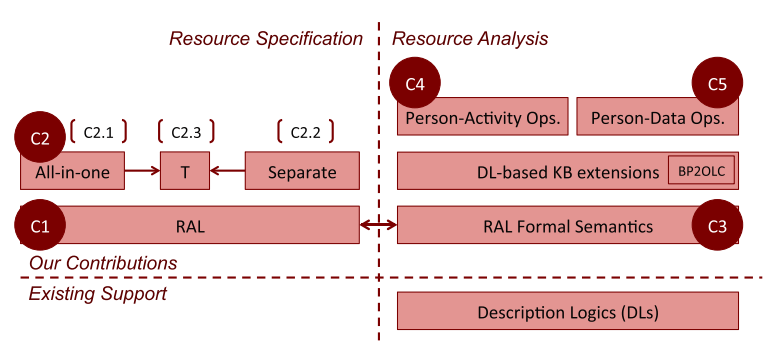

Fig. 1. Overview of the contributions. (Colors are visible in the online version of the article; http://dx.doi.org/10.3233/AIC-150656.)

The first contribution $(\mathrm{C} 1)$ is the development of a new language called Resource Assignment Language (RAL) [4], which enables defining resource selection conditions in the context of BPM. Its design is based on an existing organisational metamodel [5]. Thus, RAL expressions are traceable to the organisational model of the company, as long as it considers concepts defined in it. Furthermore, RAL is very expressive, according to the same criteria used to evaluate similar approaches on resource specification.

The second contribution (C2) pursues flexibility and introduces a technique to use RAL as an extension of Business Process Model and Notation (BPMN) (C2.1), giving rise to $R A L$-aware $B P$ models; and a technique to use RAL in combination with RASCI matrices (C2.2). Furthermore, a procedure to automatically shift from the RASCI-based approach to the BPMN-based approach in a seamless way, was developed (C2.3).

The third contribution (C3) defines formal semantics for RAL based on Description Logics (DLs) [3]. This provides the RAL expressions with precise meaning, and eases the automated extraction of information from RAL-aware BP models.

Next, relying on RAL semantics, an approach to automatically analyse how resources are managed in a BP is introduced, specifically automated support for the eleven analysis operations identified. The resolution of the person-activity operations (C4) enables answering questions such as "who can be involved in activity Sign Authorisation?", "can anybody in the company take part in all the activities of the Trip Management process?", or "is there any person indispensable for the completion of that BP?", in an automatic way. The resolution of the person-data operations (C5) provides an automatic answer to questions such as "who can read the Authorisation Form?", or "who is allowed to update the User Properties file?". As an intermediate step, some procedures and extensions of the DL-based knowledge base created to define RAL semantics were developed in order to prepare it for auto- mated operation resolution. It implied considering perspectives such as control flow (e.g., behavioural relationships between activities) and data flow (e.g., data states and transitions) for the automation of certain operations. Then, a reference implementation based on DLs was developed to enable the execution of all the operations at DT and at RT, by expressing the analysis operations in terms of standard DL reasoning operations, which are implemented by existing DL reasoners.

Support for all the contributions was implemented in CRISTAL [2], and some results were also validated in the scope of a project with a multinational organisation, in which the applicability of the automated analysis to check conformance with business rules was proven.

\section{Conclusions}

The outcome of this work is a set of traceable, expressive and flexible techniques to assign resources to BPs, and DL-based mechanisms to automate a number of resource analysis operations at DT and at RT. This bridges the gaps found in current approaches.

\section{Acknowledgements}

This work was also possible thanks to A. RuizCortés and M. Resinas, and it was partially supported by the Spanish and the Andalusian R\&D\&I programmes (P12-TIC-1867, TIN2012-32273, TIC-5906, TIN2009-07366, P07-TIC-2533).

\section{References}

[1] C. Cabanillas, Enhancing the management of resource-aware business processes, PhD thesis, University of Seville, 2012, available at: http://goo.gl/FpjDcs.

[2] C. Cabanillas, A. del Río-Ortega, M. Resinas and A. RuizCortés, CRISTAL: Collection of Resource-centrIc Supporting Tools And Languages, in: BPM Demos, 2012, pp. 51-56.

[3] C. Cabanillas, M. Resinas and A. Ruiz-Cortés, Defining and analysing resource assignments in business processes with RAL, in: ICSOC, 2011, pp. 477-486.

[4] C. Cabanillas, M. Resinas and A. Ruiz-Cortés, RAL: A highlevel user-oriented resource assignment language for business processes, in: BPM Workshops (BPD), 2011, pp. 50-61.

[5] N. Russell, A.H.M. ter Hofstede, D. Edmond and W.M.P. van der Aalst, Workflow resource patterns, Technical report, TU/e Eindhoven, 2004. 